\title{
MODEL FOR DESCRIBING EMISSION CHARACTERISTICS OF ELECTRON-BEAM EVAPORATION SOURCES
}

\author{
MIKLÓS SZILÁGYI \\ Department of Electronics Technology, Technical University of Budapest, H-1521 Budapest, Hungary
}

(Received July 12, 1978)

\begin{abstract}
The experimental data for the anistropic component of the angular distribution of electron-beam evaporation sources are usually described in terms of the $\cos ^{n} \theta$ dependence. In this paper a simple model for calculating angular distributions is presented. The generally used dependence appears as a particular case in the model.
\end{abstract}

\section{INTRODUCTION}

In vacuum deposition of thin films, the uniformity of the thickness is an important, extensively studied problem. This uniformity is required for correct operation of the devices for which the thin films are used but the efficiency of the production often strongly depends on how large the useful area is for a given permitted maximum thickness deviation. Some techniques such as metallization of semiconductor devices produce steps on the substrate. For correct operation of these devices it is required that the deposited film thickness in the steps is approximately the same as in the planar surfaces.

For depositing constant uniformity films, several methods have been developed. The substrate may be rotated either simply or by planetary motion, ${ }^{1}$ sometimes using special shields. ${ }^{2}$ The thickness distribution has been calculated for various geometries. ${ }^{1,3}$ The exact mathematical treatment of Valeiev and Avdeiev $^{4}$ makes it possible to calculate thickness distributions for any substrate shape and geometry for any kind of target-movements even in the case of multiple evaporation sources used simultaneously.

To calculate the thickness distribution, the angular dependence of the vapour emitted from the source, i.e. the angular characteristics, must be known. According to Knudsen ${ }^{5}$ the angular characteristics of small resistance-heated sources may be described by the following cosine dependence.

$$
i(\theta) \mathrm{d} \omega=\cos \theta \mathrm{d} \omega
$$

where $i(\theta) \mathrm{d} \omega$ is the amount of material emitted within the solid angle $d \omega$ in the direction $\theta$. Eq. 1 is normalized such a way that, in the direction of maximum intensity in a unit solid angle, the emitted amount of material is unity. Angular characteristics will be normalized this way in this paper.

Eq. 1 is experimentally found useful for conventional resistance-heated sources but not for electronbeam heated ones. The angular characteristics of electron-beam heated aluminium sources has been described by Graper ${ }^{6}$ using the following empirical equation

$$
i(\theta) \mathrm{d} \omega=\left[(1-A) \cos ^{n} \theta+A\right] \mathrm{d} \omega
$$

where $A$ is an empirical factor termed by Graper $^{6}$ as the isotropic component which, according to holechamber measurements, is interpreted as a consequence of the high density virtual source formed above the real one. The isotropic coefficient is found to be rather small (about 0.1) indicating that the contribution of the virtual source in the whole process is small. Exponent $n$ in Eq. 2 is found to be between 2 and 6 depending on the rate of evaporation (input power) and it increases with increasing rate of evaporation. According to Eq. 2 most of the evaporated material is more angular dependent than would follow from the simple cosine law. Angular characteristics like those of Eq. 2 were also found by Valeiev et $\mathrm{al}^{7}$ in a somewhat different arrangement and by some studies in Hungary ${ }^{8}$ for other materials.

The aim of this paper is to present a model based on the real physical system by which the experimentally found angular characteristics of electron-beam evaporating sources may be interpreted, especially the strongly angular dependent part, which has not yet been properly described. 


\section{MODEL OF THE ELECTRON-BEAM SOURCE}

Where the electron-beam hits the target a crater-like curved surface is formed, as shown in Figure 1. The temperature of this crater-surface and consequently the rate of evaporation depends on the coordinates. By assuming that the surface is cylindrically symmetrical and that the evaporation proceeds according to Eq. 1 (Knudsen equation), the intensity emitted in the solid angle $d \omega$ is

$$
\Delta i \mathrm{~d} \omega=k g(r) \cos (\mathbf{R}, \mathbf{n}) \Delta F \mathrm{~d} \omega
$$

where $k$ is a factor for normalization, $g(r)$ is the intensity emitted normally from a surface element $\Delta F$ which is located at a distance $r$ from axis $Z, \mathbf{R}$ is a vector directed in the direction $\theta$, and $\mathbf{n}$ is the normal vector to the surface element $\Delta F$.

By introducing cylindrical coordinates

$$
z=f(r, \phi)
$$

the surface element is expressed as

$$
\Delta F=r \Delta \phi\left(\Delta r^{2}+\Delta f^{2}\right)^{1 / 2}=\left[1+\left(\frac{\partial f}{\partial r}\right)^{2}\right]^{1 / 2} \Delta r \Delta \phi
$$

Assuming that vector $\mathbf{R}$ is in the $X Z$ plane, by which the generality of the treatment is not affected,

$$
\begin{aligned}
\cos (\mathbf{R}, \mathbf{n})= & \frac{\partial f}{\partial r} \cos \phi\left[1+\left(\frac{\partial f}{\partial r}\right)^{2}\right]^{-1 / 2} \sin \theta+ \\
& {\left[1+\left(\frac{\partial f}{\partial r}\right)^{2}\right]^{-1 / 2} \cos \theta }
\end{aligned}
$$

According to Eqs 3 and 6 the intensity emitted from the space element $\Delta F$ in a direction $\theta$ is

$$
\Delta i \mathrm{~d} \omega=k g(r)\left(\frac{\partial f}{\partial r} \cos \phi \sin \theta+\cos \theta\right) r \Delta r \Delta \phi \mathrm{d} \omega
$$

The total intensity is given by

$$
i(\theta) \mathrm{d} \omega=k \int_{0}^{2 \pi} \int_{0}^{\infty} g(r)\left[\frac{\partial f}{\partial r} \cos \phi \sin \theta+\cos \theta\right] r \mathrm{~d} r \mathrm{~d} \phi \mathrm{d} \omega
$$

The integral of the $\phi$ dependent part in the brackets is zero because of its symmetry; consequently

$$
i(\theta) \mathrm{d} \omega=k \int_{0}^{2 \pi} \int_{0}^{\infty} g(r) r \cos \theta \mathrm{d} r \mathrm{~d} \phi \mathrm{d} \omega
$$

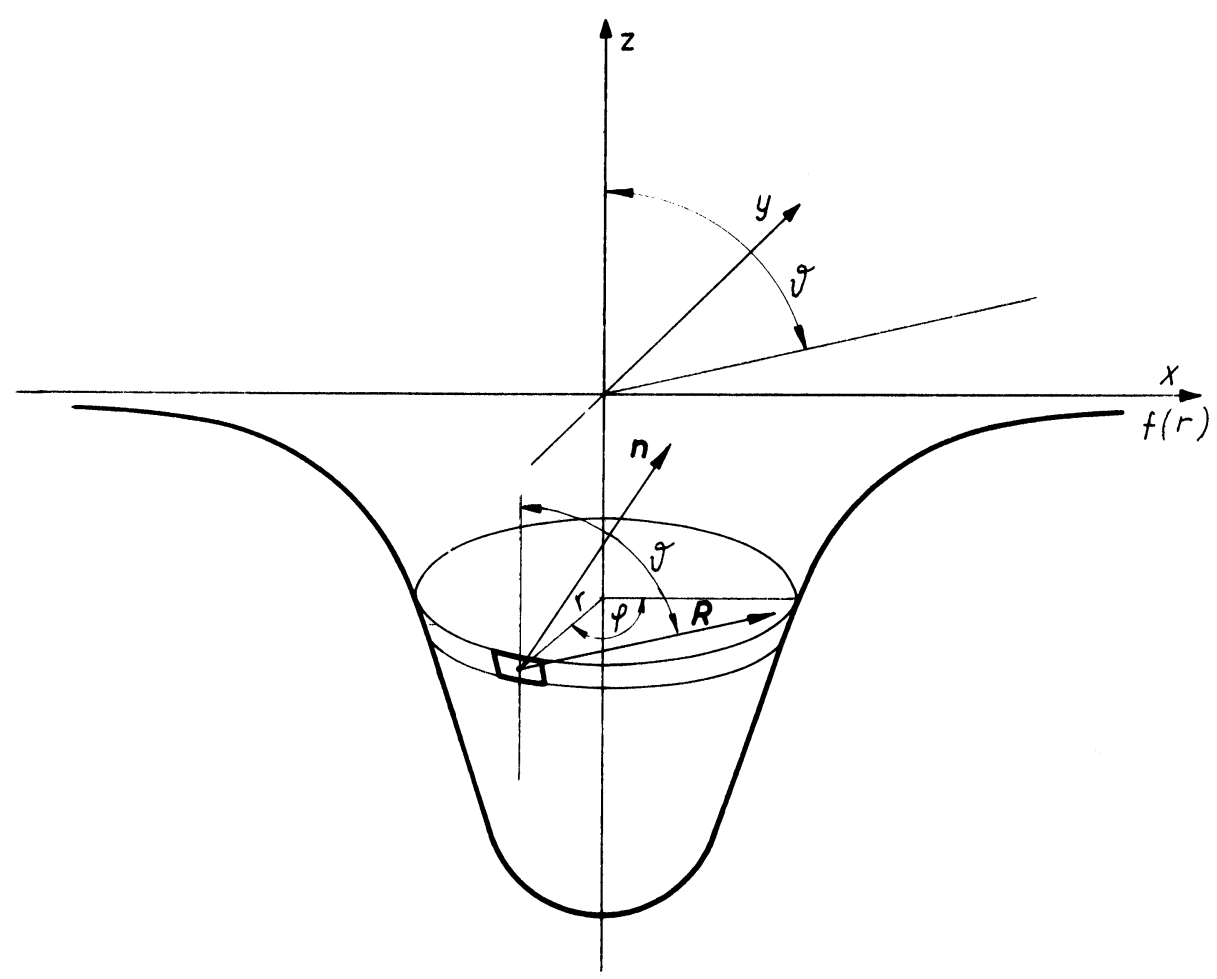

FIGURE 1 Surface of the target hit by the electron beam 


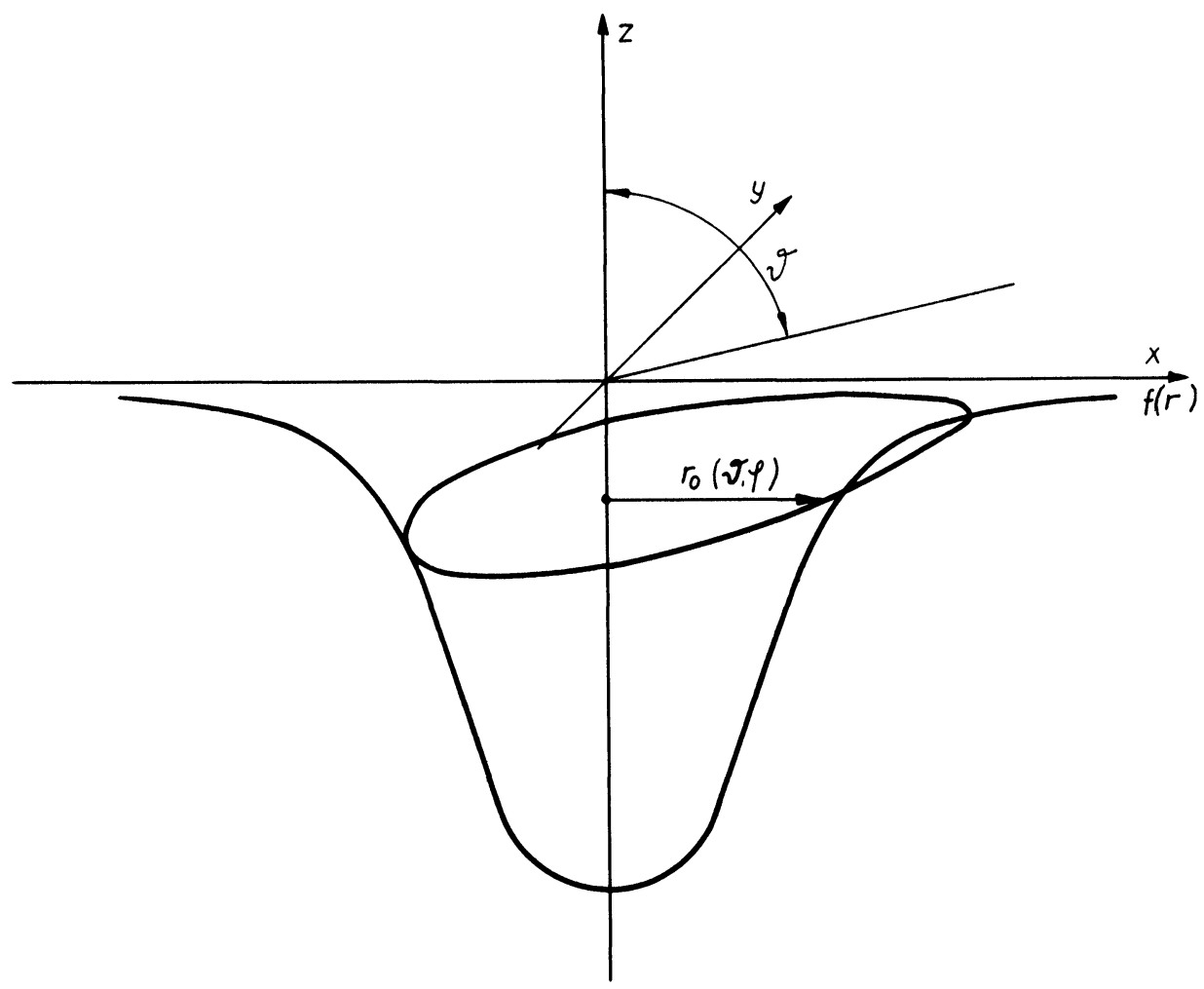

FIGURE 2 Shadow limit curve.

Eq. 9 would indicate that in the present case of nonuniform evaporation from a curved surface the cosine law (Eq. 1) should apply. As shown in Figure 2 , however, a part of the conic surface does not take part in the emission because of shielding. This is accounted for by changing the lower limit of the integration with $r$ to $r_{0}(\theta, \phi)$ which is the limiting curve of the shielding effect.

$$
i(\theta) \mathrm{d} \omega=k \int_{0}^{2 \pi} \int_{r_{0}(\theta, \phi)}^{\infty} g(r) r \cos \theta \mathrm{d} r \mathrm{~d} \phi \mathrm{d} \omega
$$

The integral of the first term in Eq. 8 is zero in this case too, because $r_{0}(\theta, \phi)$ is symmetrical with respect to the $X Z$-plane.

Angular characteristics can be calculated according to Eq. 2. For this $g(r)$ and $f(r)$ must be known. From the physical picture some general properties of these functions follow directly. Both should be single valued, monotonic, bounded and approximate to zero if $r \rightarrow \infty ; g(r)$ is a decreasing, $f(r)$ an increasing function.

Assumptions about explicit forms of these functions will be given later. The following points are, however, of note.
1) As $r_{0}(\theta, \phi)$ is a monotonically increasing function of $\theta$ the corresponding integral in Eq. 10 decreases indicating that the angular dependence is steeper than that described Eq. 1.

2) If shielding is not effective up to a certain angle $\theta_{0}$, i.e. if at all parts of the surface

$$
\left|\frac{\partial z}{\partial x}\right|_{\max }=\left|\frac{\partial z}{\partial y}\right|_{\max }<\operatorname{cotan} \theta_{0}
$$

then the angular characteristic is that of Eq. 1 in the angular range $\left(-\theta_{0}, \theta_{0}\right)$.

3) It would appear that this model does not take into consideration the particles which leave the surface indirectly, i.e. after reflection. According to experimental results of Knudsen, ${ }^{9}$ however, the molecular beam is scattered diffusely on the surface. Correspondingly, the emission characteristics of the secondary (or multiple) scattered particles should also follow the cosine law. This implies that the shape of the $g(r)$ function is affected by this effect only.

4) The functions $g(r)$ and $f(r)$ which are necessary for actual determination of angular characteristics, cannot be exactly determined because 
they are dependent in a complex way on the intensity distribution of the focussed electronbeam, on the rate of evaporation and on material properties. No experimental ways are known at present for determining these functions with sufficient accuracy.

Consequently it is attempted to introduce some realistic functions which fulfil the general properties discussed above in order to describe angular characteristics in a concrete form.

\section{MODEL WITH REALISTIC FUNCTIONS}

It is expected that the density distribution of the electrons hitting the target is approximated by the Gaussian function. The heating-up of the target and the rate of evaporation are influenced by many parameters but the resulting evaporating rate versus coordinate relation may also be assumed as Gaussian: 10

$$
g(r)=C \exp \left(-\frac{r^{2}}{D^{2}}\right)
$$

where $C$ and $D$ are constants.

Using these assumptions the integration with $r$ in Eq. 10 can be performed to result in

$$
i(\theta) \mathrm{d} \omega=k \int_{0}^{2 \pi} \exp \left[-\frac{r_{0}^{2}(\theta, \phi)}{D^{2}}\right] \mathrm{d} \phi \cos \theta \mathrm{d} \omega
$$

The spatial curve $r_{0}(\theta, \phi)$ can, in principle, be determined by knowing the equation of the surface $f(r)$, but this is rather complicated. It seems more useful therefore to introduce assumptions about $r_{0}(\theta, \phi)$ directly instead of $f(r)$.

For real $f(r)$ surfaces at a given angle $|\theta|<\pi / 2$ the 'shadow' limiting $r_{0}(\theta, \phi)$ curve is a positive, continuous and bounded function of $\phi$. According to the integral mean-value theorem, the integral with $\phi$ may be substituted by $2 \pi \exp \left[-r_{0}^{2}\left(\theta, \phi^{*}\right) / D^{2}\right]$ where $\phi^{*}$ is a value of $\phi$ within the range $(0,2 \pi)$. According to this, and introducing $r_{0}(\theta)=r_{0}\left(\theta, \phi^{*}\right)$, the angular characteristic is obtained in the normalized form as

$$
i(\theta) \mathrm{d} \omega=\exp \left[-r_{0}^{2}(\theta) / D^{2}\right] \cos \theta \mathrm{d} \omega
$$

According to point 2 of the previous section, $r_{0}(\theta)$ becomes zero in a certain range $0<|\theta|<\theta_{0}$. Experimental results ${ }^{6,7,8}$ show, however, that for usual arrangements and evaporation rates $\theta_{0}$ is small i.e. the 'wall' of the $f(r)$ surface is 'steep'. Thus an $r(\theta)$ curve should be chosen which would shrink into a point at $\theta=0$ and $r(\theta)>0$ if $|\theta|>0$. On the other hand it is evident from Figure 2 that in the limit of $\theta=\pi / 2, r(\theta)$ should tend to infinity. The anisotropic component of the empirical Graper equation is obtained by using

$$
r_{0}(\theta)=D[-(n-1) \ln (\cos \theta)]^{1 / 2}
$$

in Eq. 13. This function fulfils the criteria for limits $\theta=0$ and $\theta=\pi / 2$ discussed above. Parameter $D(n-1)^{1 / 2}$ characterizes the slope of the $r_{0}(\theta)$ and the evaporating surface $f(r)$. It is interesting to note that some other functions such as constant $\tan \theta$ or constant.sin $\theta$ also result in similar angular characteristics which can be well fitted with the experimental data.

\section{ACKNOWLEDGEMENTS}

The author wishes to express his gratitude to Gy. Vago for calling attention to the problem and to A. Ambrozy for his valuable advices and discussions.

\section{REFERENCES}

1. K. H. Behrndt, "Thickness Distribution and Step Coverage in a New Planetary Substrate Holder Geometry," J. Vac. Sci. and Techn., 9, 2 (1972) p. 995.

2. L. Nulchis, G. Ripka, M. Szilagyi, "Vekonyreteg ellenallasok pontossaganak egyes kerdesei," Finommechanika, 12, 10, p. 300 (1973).

3. B. S. Ramprasad, T. S. Radha, "Uniformity of Film Thickness on Rotating Planetary Planar Substrates," Thin Solid Films, 15, 1, p. 55 (1973).

4. A. S. Valeiev, E. V. Avdeiev, "Rastsot profilia sloia skondensirovanogo v vakuume na relefnuiu podlozku," In "Mikroelektronika" Moscow, "Sovietskoie radio" 8. (1975) p. 164.

5. M. Knudsen, "Das Cosinusgesetz in der kinetischen Gastheorie," Ann. Physik., 48, p. 471 (1930).

6. E. B. Graper, "Distribution and Apparent Source Geometry of Electron-Beam-Heated Evaporation Sources," J. Vac. Sci. and Techn., 10, 1. p. 100 (1973).

7. A. S. Valeiev, V. D. Somash, "Emisionie Kharakteristiki isparitelei aluminiia s resistivnim i elektronim nagrevam," Elektronnaia Tekhnika, Seriia 3 (Mikroelektronika) 2 (68) p. 83 (1977).

8. B. Szikora, "Elektronsugaras parologtato forras iranykarakterisztikajanak vizsgalata," Diplomwork, BME, Bp. (1976).

9. M. Knudsen, "Die Gesetze der Molekulastromung und der inneren Reibungstromung der Gase durch Rohren," Ann. Physik, 28, p. 75 (1909).

10. Gy. Vago (private communication). 

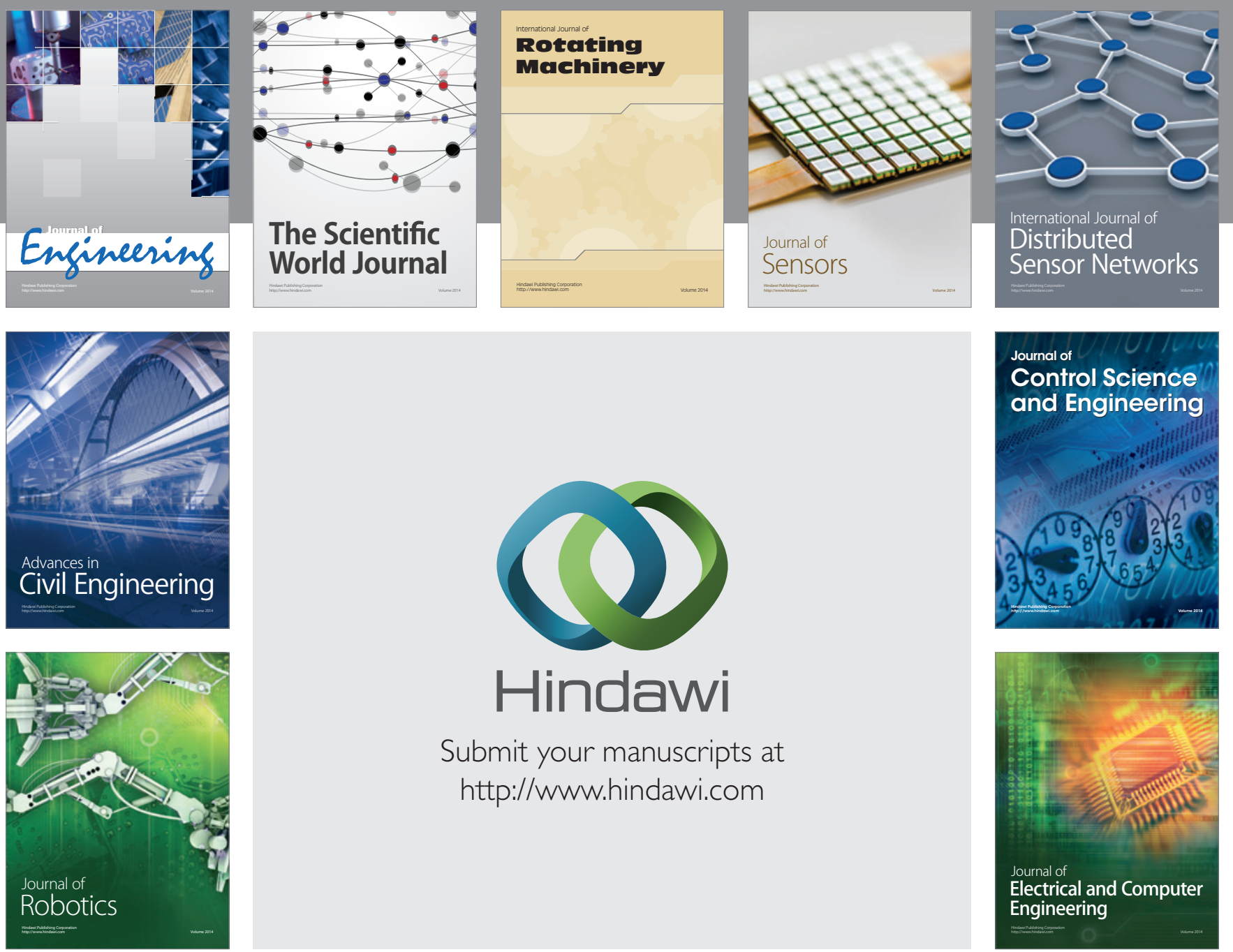

Submit your manuscripts at

http://www.hindawi.com
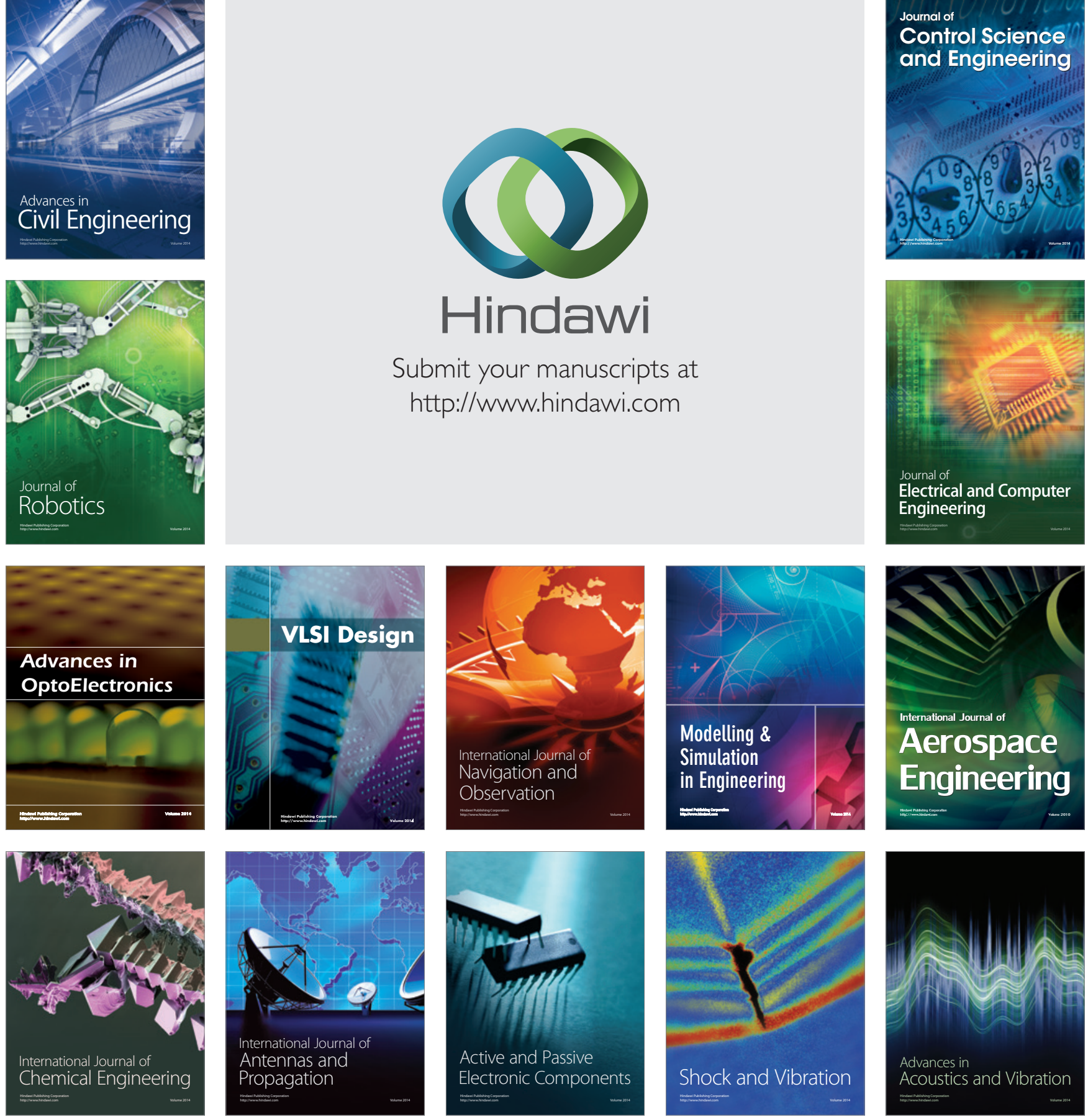